\title{
On the Organizing Role of Nonmuscular Forces During Performance of a Giant Circle in Gymnastics
}

\author{
Violaine Sevrez, Guillaume Rao, Eric Berton, and Reinoud J. Bootsma \\ Aix-Marseille Université
}

\begin{abstract}
Five elite gymnasts performed giant circles on the high bar under different conditions of loading (without and with 6-kg loads attached to the shoulders, waist or ankles). Comparing the gymnasts' kinematic pattern of movement with that of a triple-pendulum moving under the sole influence of nonmuscular forces revealed qualitative similarities, including the adoption of an arched position during the downswing and a piked position during the upswing. The structuring role of nonmuscular forces in the organization of movement was further reinforced by the results of an inverse dynamics analysis, assessing the contributions of gravitational, inertial and muscular components to the net joint torques. Adding loads at the level of the shoulders, waist or ankles systematically influenced movement kinematics and net joint torques. However, with the loads attached at the level of the shoulders or waist, the load-induced changes in gravitational and inertial torques provided the required increase in net joint torque, thereby allowing the muscular torques to remain unchanged. With the loads attached at the level of the ankles, this was no longer the case and the gymnasts increased the muscular torques at the shoulder and hip joints. Together, these results demonstrate that expert gymnasts skillfully exploit the operative nonmuscular forces, employing muscle force only in the capacity of complementary forces needed to perform the task.
\end{abstract}

Keywords: open-chain movement, simulation, additional loads, coordination

When performing a giant circle on the high bar, the gymnast departs from a handstand position above the bar and fully rotates around it, without ever releasing the bar. Because of the friction encountered, the kinetic energy gained during the downswing is not sufficient to allow a passive gymnast to swing back up to the handstand position. Sevrez et al. (2009) recently demonstrated that gymnasts solve this problem by behaving as a pendulum of variable length. By regulating the distance between their body center of mass and the axis of rotation (i.e., the bar), the gymnast effectively controls the flow of energy through the system: energy is dissipated when the pendulum lengthens and energy is inserted when the pendulum shortens (Stilling \& Szyszkowski, 2002). Notwithstanding the power of this kind of model to capture the essential features of the function subserved - thereby providing a solid foundation for understanding what the gymnast is doing (cf. Sevrez et al. (2009) - it does not address the issue of how the gymnast implements the required variations in pendulum length.

In classical variable-length pendulums, such as the Botafumeiro (the famous giant censer of the Santiago de

Violaine Sevrez (Corresponding Author), Guillaume Rao, Eric Berton, and Reinoud J. Bootsma are with UMR 7287 ISM Marey, Aix-Marseille Université, Marseille Cedex 9, France.
Compostela cathedral in northwestern Spain), length variations are accomplished by sliding the suspended mass toward and away from the axis of rotation at particular phases of the pendulum oscillation. In the gymnast's case, however, such length variations can only be brought about through flexion and/or extension of one or more joints.

Control of such a compound pendulum presents two significant complicating factors as compared with a classical variable-length pendulum. In the first place, a given pendulum length can be achieved through many different joint configurations. Sevrez et al. (2009) demonstrated that execution of a giant circle implies significant motion at both the shoulder and hip joints. This raises the issue of the mechanisms underlying the emergence of one particular pattern of movement rather than another. In the second place, intersegmental dynamics come to the fore, with each body segment of such a connected multibody system affecting the motion of all the other segments in the chain (Hoy \& Zernicke, 1986; Hunter et al., 2004). Indeed, inertial torques have been shown to play a significant role in the intersegmental dynamics of multijoint movements (Hollerbach \& Flash, 1982), especially so in "free-segment" motion (that is, when the distal end of the distal link is not restrained; Phillips et al., 1983). Gravitational torques have also been reported to exert substantial effects during vertical multijoint movements (Kodek \& Munih, 2003). With the giant circle being a free-segment movement involving two joints in the vertical plane, such 
nonmuscular torques may thus be expected to have prominent effects during movement execution.

If, as Bernstein (1967) contends, "the secret of coordination lies not only in not wasting superfluous force in extinguishing reactive phenomena but, on the contrary, in employing the latter in such a way as to employ active muscle forces only in the capacity of complementary forces" (p. 109), then we may expect the operative nonmuscular torques to play a structuring role in determining the pattern of movement.

With the aim to further our understanding of the mechanisms underlying the emergence of a particular pattern of movement in a particular context, the current study examined the giant circle in two successive steps. First, to qualitatively examine the organizing influences of the constraints provided by passive mechanical forces, the movement pattern produced by gymnasts actively performing the task was compared with the movement pattern emerging under the sole influence of nonmuscular torques. A triple-pendulum simulation, based on the gymnasts' anthropometrics, provided the latter. Second, as loading changes the mechanical characteristics of the gymnast (thereby further complicating the force environment and possibly implying adaptation of muscular torques needed to fulfill the task, cf. Browning et al. 2007), we analyzed the effects of additional weights placed at the level of the shoulders, waist or ankles. With the goal to gain insight into the respective contribution of passive and active components to the net torques produced, this analysis concerned both the movement patterns produced and, through inverse dynamics, the gravitational, interactive and muscular components of the net joint torques of gymnasts performing giant circles.

\section{Methods}

\section{Experimentation}

Five elite senior male gymnasts without any history of joint neuromuscular disease volunteered for participation in the experiment. Their ages ranged from 17 to 20 years (mean $19.0 \pm 1.2$ years), their standing height from 1.63 to $1.71 \mathrm{~m}$ (mean $1.64 \pm 0.06 \mathrm{~m}$ ), and their body mass from 49.0 to $65.0 \mathrm{~kg}$ (mean $55.8 \pm 7.0 \mathrm{~kg}$ ). Participants gave their informed consent before inclusion in the study, which was approved by the local ethics committee.

Gymnasts performed backward giant circles on the high bar under an (unloaded) control condition and three conditions with additional loads (6 kg total) symmetrically placed on the upper arms near the shoulder joints, the waist, or the lower legs near the ankles. They were instructed to perform the backward giant circles with respect to the code of points of the International Gymnastics Federation (2006). The gymnasts were instructed to conclude each swing in a vertical handstand position with minimal rotational velocity before initiating the next. After a few trials performed to allow adaptation to the experimental situation, participants were requested to perform seven subsequent giant circles in each experimental condition. No temporal constraints were imposed during the session.

Two-dimensional joints locations were recorded at $150 \mathrm{~Hz}$ using a Basler video camera placed perpendicularly to the plane of motion. Six markers were attached to the subjects' skin over the following right side joints: wrist, elbow, shoulder, hip, knee, and ankle (see Zatsiorsky, 2002, for the bony landmarks).

To examine stable performance, of the seven giant circles recorded only giant circles two to six were analyzed. Marker locations were manually digitized and global 2D coordinates were computed using SIMI motion software. The position data were low-pass filtered with a zero time lag fourth-order Butterworth filter at a net cutoff frequency of $6 \mathrm{~Hz}$. Segmental masses and moment of inertia of individual participants were estimated using the anthropometric tables of Zatsiorsky and Seluyanov (1983).

The center-of-mass trajectory was obtained by computing its location for each frame of the cycle. Following Yeadon and Hiley (2000), body orientation was defined as the angle between the vertical and a line from the bar to the body center of mass (BCM). For each experimental condition, the body orientation angle $(\theta)$, shoulder angle, and hip angle were estimated and time-differentiated to obtain angular velocities and accelerations. All of the angles were set to zero in the handstand position above the bar and positive values represented flexion for the shoulder and hip joints.

Bilateral symmetry was assumed and a threesegments, three-degrees-of-freedom, planar model of the body was used to estimate net joint forces and torques during performance of the giant circles under each of the loading conditions. The model incorporated a headtrunk segment, a lower limb segment and an upper limb segment. The equations were formulated by using the usual assumptions of planar motion, rigid segments, frictionless joints and joint reaction forces acting through joint centers (Bresler \& Frankel, 1950). The Lagrangian formulation was used to allow estimation of the gravitational, interactive and muscular components of shoulder and hip joint torques, by considering net torques as the sum of gravitational, interactive and muscular torques.

For all dependent variables, we calculated, for each individual participant, the average over the five analyzed giant circles in each condition. Repeated-measures ANOVA were used to identify the effects of loading on the peak values of (i) shoulder and hip flexion and extension, (ii) shoulder and hip net, interaction, gravitational, and muscular torques. Tukey post hoc tests were used when significance level $(p<.05)$ was reached.

\section{Simulation}

A three-rigid-bodies (upper limb, head-trunk, and lower limb) planar model of a gymnast was created to simulate the effect of nonmuscular torques during a rotational movement around the bar. The output of the simulation consisted in the time course of the "hip" and "shoulder" joints angles. The bar was rigidly attached to a fixed polar coordinate system with the axis pointing up. The 
two vectors comprising respectively the three joint angles and the three joint angular velocities specified the state of the system. Following Cui et al. (1995), segment equations of motion were derived based on the Lagrangian formulation. The three obtained second-order differential equations were then transformed into six first-order differential equations, and a fourth-order Runge-Kutta integration algorithm was used to compute iteratively an approximation of the solution of this system of equations. The integration time step was set to $\Delta \mathrm{t}=0.006 \mathrm{~s}$ and gravity to $g=9.80665 \mathrm{~m} / \mathrm{s}^{2}$. Segment parameters were defined based on participants' mean measurements (Table 1). Friction coefficient at the bar-hand joint and joint excursion were entered into the simulation based on measurement performed during the experimental session, according to a methodology detailed hereafter.

The bar-hand friction coefficient to be included in the simulation was determined in two successive steps. We first measured experimentally the height reached by a gymnast swinging-up after having swung-down from the handstand position above the high bar while letting himself go and keeping his body as straight as possible. We then determined the value of the coefficient of friction from a simulation of a simple pendulum having the same anthropometric characteristics as the studied gymnast, by progressively increasing the coefficient of friction at the bar-hand joint until the pendulum swung back up to the same height as the gymnast. The determined coefficient $(0.15)$ was included in the simulation.

The passive effect of muscles on shoulder and hip joint excursion were included in the simulation. The passive forces were estimated as a function of the hip and shoulder joint angles using the Opensim software (Delp et al. 2007) and then fitted with potential functions used as constraints in the simulation.

\section{Results}

The pattern of movement adopted by the gymnasts while performing the task under the nonloaded control condition is represented in the form of a kinogram (Figure 1A). Maximal hip extension $\left(26.2 \pm 0.9^{\circ}\right)$ was reached at the end of the descent phase, at 158.2 $\pm 3.1^{\circ} \mathrm{BCM}$ rotation angle and maximal shoulder extension $\left(1.1 \pm 0.3^{\circ}\right)$ was reached just before the lowest point, at $175.4 \pm 2.4^{\circ}$

Table 1 Mean body segment parameters of the five elite gymnasts participating in the experiment

\begin{tabular}{lcccc}
\hline Segment & Length $(\mathbf{m})$ & Mass $(\mathbf{k g})$ & $\begin{array}{c}\text { Center of } \\
\text { Mass }(\mathbf{m})\end{array}$ & $\begin{array}{c}\text { Moments of } \\
\text { Inertia }\left(\mathbf{k g} / \mathbf{m}^{2}\right)\end{array}$ \\
\hline Upper limb & $0.519 \pm 0.020$ & $5.593 \pm 0.592$ & $0.301 \pm 0.013$ & $0.173 \pm 0.032$ \\
Trunk & $0.502 \pm 0.019$ & $28.361 \pm 3.346$ & $0.175 \pm 0.013$ & $1.107 \pm 0.180$ \\
Lower limb & $0.808 \pm 0.032$ & $21.956 \pm 3.009$ & $0.272 \pm 0.011$ & $1.442 \pm 0.312$ \\
\hline
\end{tabular}

A.

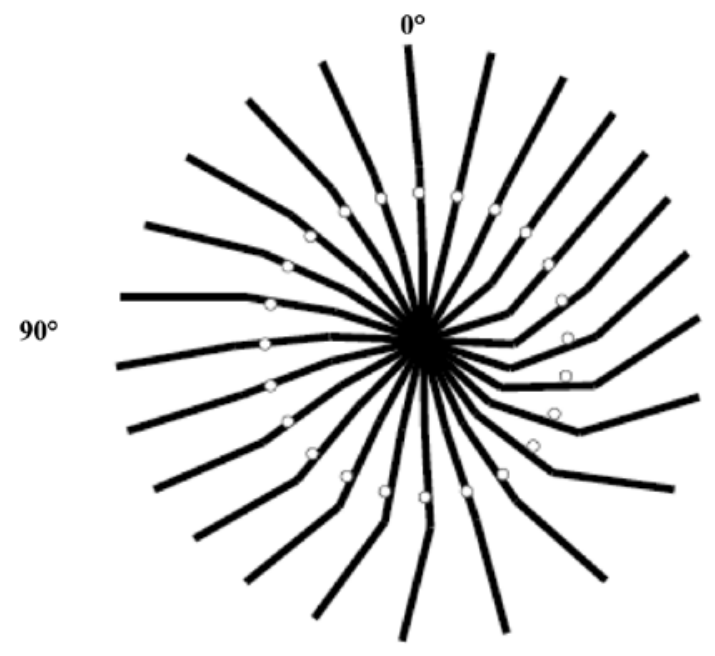

$180^{\circ}$
B.

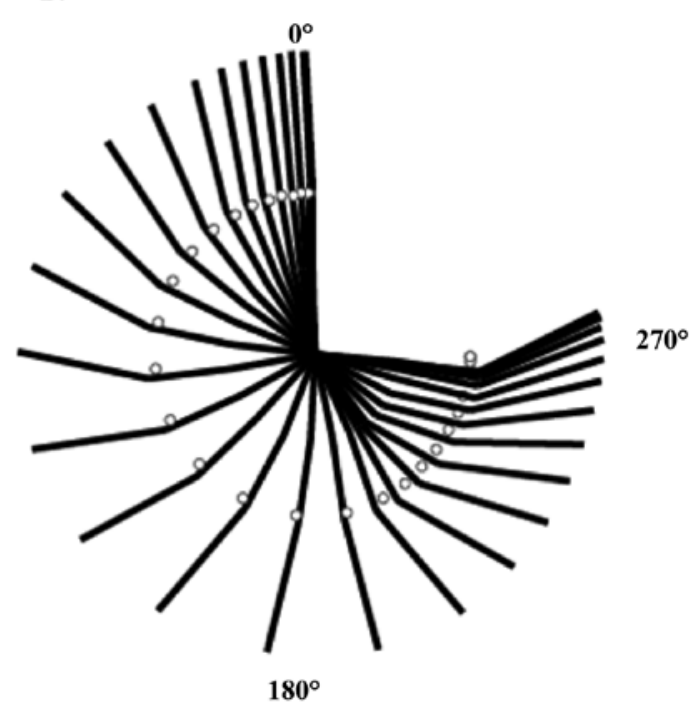

Figure 1 - Kinograms of (A) a gymnast performing a backward giant circle (counter-clockwise) on the high bar and (B) a passively falling triple pendulum with the same anthropometric parameters. Positions are represented in steps of $15^{\circ}$ of body center of masse rotation angle. White dots represent the position of the global center of mass for each frame. 
$\mathrm{BCM}$ rotation angle. During the ascent phase maximal hip flexion $\left(35.1^{\circ} \pm 2.5^{\circ}\right)$ was reached at $258 \pm 4.1^{\circ} \mathrm{BCM}$ rotation angle and maximal shoulder flexion $\left(38.9 \pm 1.9^{\circ}\right)$ was reached at $283 \pm 3.8^{\circ} \mathrm{BCM}$ rotation angle.

For comparative purposes, the motion pattern of the simulated triple pendulum (driven solely by nonmuscular torques) is also represented as a kinogram (Figure 1B). Starting from a extended position above the bar, the triple pendulum first extended during the downswing with maximal "hip" extension (about $24^{\circ}$ ) being reached at $143^{\circ} \mathrm{BCM}$ rotation angle and maximal "shoulder" extension (about $4^{\circ}$ ) being reached at $172^{\circ} \mathrm{BCM}$ rotation angle. Interestingly, under the influence of the nonmuscular torques the compound pendulum subsequently flexed during the upswing with maximal "shoulder" (about $35^{\circ}$ ) and "hip" (about $31^{\circ}$ ) flexions being reached at $263^{\circ}$ and $272^{\circ} \mathrm{BCM}$ rotation angles, respectively.

Overall, the patterns of movement of the active gymnast and the passive triple pendulum revealed striking similarities, providing a first indication of the structuring role of the nonmuscular torques.

Despite the presence of additional loads, on average representing more than $10 \%$ of the body weight, all participants were able to perform the giant circles. Corroborating the results reported in more detail by Sevrez et al. (2009) for junior female gymnasts, load location subtly but systematically influenced the timing, and to a lesser degree, the magnitude of peak joint extension and flexion for senior male gymnasts (see Figure 2). While the pattern of shoulder and hip joint motion thus changed to a certain extent over the loading conditions, it retained the qualitative characteristics described earlier.

Because loading changes the mechanical characteristics of gymnasts, inverse dynamics were used to quantify the kinetic effects produced. Figure 3 presents the evolution over body orientation angle of net torques, as well as the gravitational, interactive, and muscular components, operating at the shoulder and the hip joint when performing backward giant circles under each of the three loaded conditions (i.e., conditions with $6 \mathrm{~kg}$ attached at the level of the ankles, hips or shoulders).

The net torques (NT) were affected by the presence of loads at different locations (Figure 3). ANOVAs performed on the values of the two extension and the flexion peaks of shoulder NT revealed significant effects of load location (all $p$ 's $<.001$ ) with the three peaks being more marked with loads attached to the ankles and the second extension peak being less marked with loads attached at the level of the shoulder. Similarly, the three hip NT peaks were significantly (all $p$ 's $<.001$ ) more marked for loads attached to the ankles as compared with the other conditions.

As was to be expected, the Gravitational Torques (GT) were systematically affected by the location of the loads (Figure 3). Shoulder peak flexion and extension GTs revealed significant effects of load location ( $p$ 's $<.001)$ with both peaks being more marked for loads attached at the ankles and less marked for loads attached at the shoulders as compared with the condition with loads at the hips. At the level of the hip joint, both peak flexion and peak extension GTs were significantly ( $p$ 's $<.001)$ more pronounced in the condition with loads attached at the ankle than in the other conditions.

The location of the loads also systematically influenced the Interaction Torques (IT, Figure 3). Peak shoulder ITs revealed significant effects of load location (all $p$ 's $<$.001) with all four IT peaks being more marked with loads fixed at the ankle than for the other conditions and the first two IT peaks being less marked for the conditions with loads at the shoulder than for the other conditions. At the level of the hip, the ITs presented two extension peaks and a flexion peak. These three IT peaks revealed significant effects of load location (all $p$ 's < .001) with all of the peaks being more pronounced for loads at the ankles than for the other conditions and the flexion peak
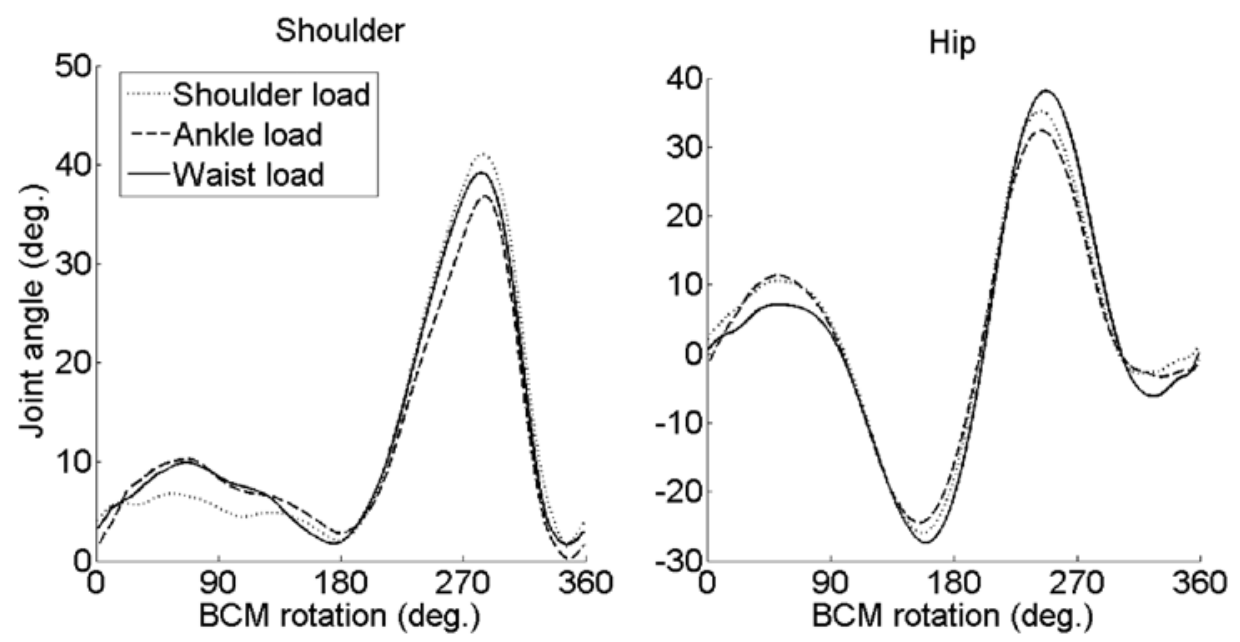

Figure 2 - Effects of adding loads at the level of the shoulders (dotted lines), waist (continuous line) and ankles (dashed) on the averaged shoulder (left panel) and hip (right panel) joint angles as a function of the body center of mass (BCM) rotation angle. 

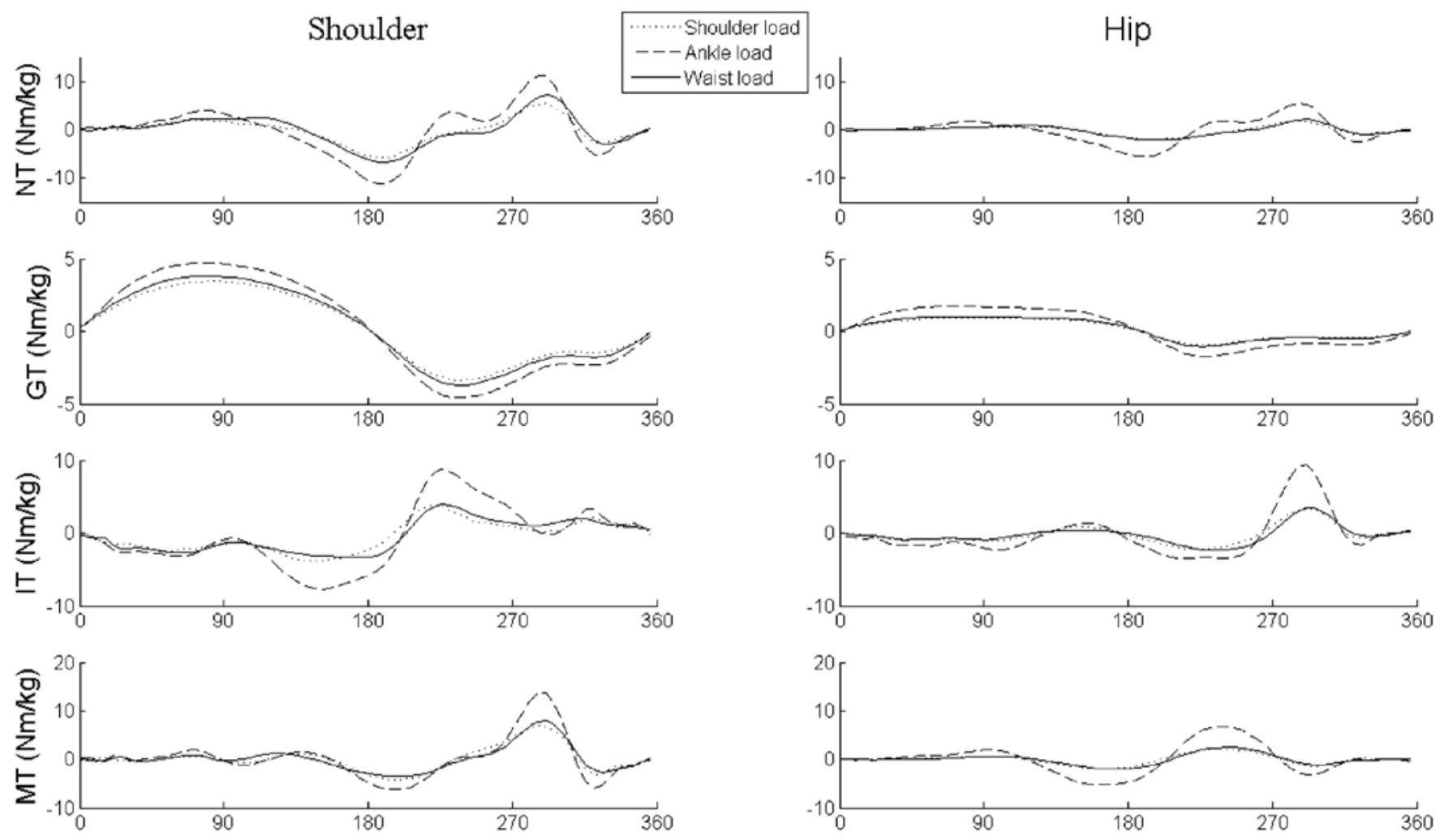

Figure 3 - Evolution of the net joint torques (NT), gravitational torques (GT), interactive torques (IT), and muscular torques (MT) for the shoulder and the hip joints as a function of the body rotation angle, averaged over subjects and trials for the condition with $6 \mathrm{~kg}$ loads attached to the shoulders (dotted line), waist (continuous line) and ankles (dashed).

being less pronounced for loads at the waist than for the other conditions.

Finally, the Muscular Torques (MT) also varied with load location (Figure 3). Both the shoulder and the hip joints demonstrated two extension peaks and one flexion peak. For both joints, these MT peaks were significantly more marked for loads attached to the ankles than for the other conditions (all p's $<.001$ ).

\section{Discussion}

In the present contribution we explored the role of gravitational and inertial torques in the organization of full-body movement. In a first step we compared the pattern of movement of gymnasts performing a giant swing on the high bar with that of a compound pendulum endowed with realistic anthropometrical and frictional characteristics. As the gymnasts, the compound pendulum demonstrated an arched position during the downswing and a piked position during the upswing. Starting from a handstand above the bar, during the downswing both revealed a systematic pattern of extension, with peak hip extension occurring before peak shoulder extension. During the upswing, again, a systematic pattern of flexion was observed, with maximal flexion of the shoulder and hip joints being reached around passage through the horizontal. This similitude between the behaviors of a compound pendulum and a gymnast performing a giant circle confirms the reported significance of intersegmental dynamics in determining the motions of free (Phillips et al., 1983) multisegment (Hollerbach \& Flash, 1982) systems during movements performed in the vertical plan (Kodek \& Munih, 2003). The coherence between the movement pattern adopted by the gymnast and the behavior of the compound pendulum suggests that gymnasts do not seek to compensate for nonmuscular forces, but rather seek to exploit them.

In a second step, we examined the effects of adding loads to the gymnasts' shoulder, hip or ankle joints. The additional nonmuscular forces generated by the presence of such loads act as an external disturbance that further complicates the force environment in which the action takes place. Given that all gymnasts were able to perform the backward giant circle under all loading conditions, we must conclude that, under each condition, they succeeded in inserting the required energy at the right place and time to deal with the particulars of the situation. As a kinematic analysis only quantifies the resultant movement, an inverse dynamics analysis was performed to pinpoint the variations in the constituent components of joint torques. Browning 
et al. (2007) suggested that the location of an additional load affects the muscular torques produced. In line with this finding, our results revealed two different relationships between muscular and nonmuscular torques depending on the location of the additional loads. With the loads attached to the shoulder or waist the induced changes in IT and GT generated the changes in NT necessary to succeed the task and MT remained similar to that observed during the unloaded condition. However, with the loads attached to the ankles the changes induced in IT and GT did not provide the NT necessary to succeed the task and the gymnasts increased the amount of MT produced.

Taking these two sets of results together, our study indicates that the dynamics of the three main phases of a giant swing may be explained as follow: (I) during the downswing, the gravitational and inertial torques generate an extension of the hip and shoulder joints, leading to an arched attitude of the body, (2) during the first half of the upswing, reversed gravitational torques and large inertial torques generate flexion at both joints, and (3) during the remaining of the upswing, muscle torques not only act to complement the acceleration of the more distal segments (particularly the lower-limb), but also extend both joints to attain the handstand position above the bar in an extended position. As highlighted by Hoy and Zernicke (1986), the details of the segmental dynamics complement these basic principles of control for multijoint movement and may explain the respective contribution of nonmuscular vs. muscular torques to the NT. For example, during the down-swing, the amplitudes of joint excursion, velocity, and acceleration increased in magnitude from proximal (shoulder) to distal (hip). In contrast to these kinematic relationships, the maxima1 value of net torques at the hip was significantly smaller than the (large) net torque at the shoulder joint.

The current analysis demonstrates how muscular torques are coordinated with nonmuscular torques during performance of the giant circle on the high bar in gymnastics. Just as the results of Hoy and Zernicke's (1986) experiment on the paw-shake response in adult spinal cats, our results are consistent with the principle espoused by Bernstein (1967) that the organization of movement is based on taking advantage of the operative nonmuscular torques, rather than seeking to compensate for them.

At a more practical level, our findings have implications for using additional loads for strength conditioning programs. Indeed, working with proximal loads should not be expected to enhance the force production capacities in the shoulder and hip joint muscles. Soliciting greater muscular torques by working with distal loads, on the other hand, should be expected to be beneficial.

\section{References}

Bernstein, N. (1967). The Coordination and Regulation of Movements. Oxford: Pergamon Press.

Bresler, B., \& Frankel, J.P. (1950). The forces and moments in the leg during level walking. Transactions of the American Society of Mechanical Engineers, 72, 27-36.

Browning, R.C., Modica, J.R., Kram, R., \& Goswami, A. (2007). The effects of adding mass to the legs on the energetics and biomechanics of walking. Medicine and Science in Sports and Exercise, 39(3), 515-525.

Cui, K., Haque, I., \& Thirumalai, M. (1995). On configurations of symbolic equations of motion for rigid multibody systems. Mechanism and Machine Theory, 30(8), 1149-1170.

Delp, S.L., Anderson, F.C., Arnold, A.S., Loan, P., Habib, A., John, C., et al. (2007). OpenSim: Open-source software to create and analyze dynamic simulations of movement. IEEE Transactions on Bio-Medical Engineering, 54, 1940-1950.

Hollerbach, J.M., \& Flash, T. (1982). Dynamic Interactions Between Limb Segments During Planar Arm Movement. Biological Cybernetics, 44, 67-77.

Hoy, M.G., \& Zernicke, R.F. (1986). The role of intersegmental dynamics during rapid limb oscillations. Journal of Biomechanics, 19(10), 867-877.

Hunter, J.P., Marshall, R.N., \& McNair, P.J. (2004). Segmentinteraction analysis of the stance limb in sprint running. Journal of Biomechanics, 37, 1439-1446.

International Gymnastics Federation. (2006). Code of points for men's artistic gymnastics competitions. http://www. fedintgym.com/rules/.

Kodek, T., \& Munih, M. (2003). An analysis of static and dynamic joint torques in elbow flexion-extension movements. Simulation Modelling Practice and Theory, 11(3), 297-311.

Phillips, S.J., Roberts, E.M., \& Huang, T.C. (1983). Quantification of intersegmental reactions during rapid swing motion. Journal of Biomechanics, 16(6), 411-417.

Sevrez, V., Berton, E., Rao, G., \& Bootsma, R.J. (2009). Regulation of pendulum length as a control mechanism in performing the backward giant circle in gymnastics. Human Movement Science, 28, 250-262.

Stilling, D.S.D., \& Szyszkowski, W. (2002). Controlling angular oscillations through mass reconfiguration: A variable length pendulum case. International Journal of Non-linear Mechanics, 37, 89-99.

Yeadon, M.R., \& Hiley, M.J. (2000). The mechanics of the backward giant circle on the high bar. Human Movement Science, 19, 153-173.

Zatsiorsky, V. (2002). Kinetics of human motion. Champaign, IL: Human Kinetics.

Zatsiorsky, V., \& Seluyanov, V. (1983). The mass and inertia characteristics of main segments of the human body. In $\mathrm{H}$. Matsui \& K. Kobayashi (Eds.), Biomechanics VIII-B (pp. 1152-1159). Champaign, IL: Human Kinetics. 